Excellent Woman Researcher Award of The Electrochemical Society of Japan

\title{
Development of a Molecular Recognition Electrode and Investigation of a Biomolecular Application in Non-Aqueous Media -Electrochemical Detection of Uremia-Related Substances Excreted via ATP-Binding Cassette Transporter G2-
}

\author{
Kyoko FUJITA, ${ }^{*, \S}$ [1] Misaki SEKIDO, Kohei KANNO, Kio HATAE, and Kimiyoshi ICHIDA
}

Department of Pathophysiology, Tokyo University of Pharmacy and Life Sciences, 1432-1 Horinouchi, Hachioji, Tokyo 192-0392, Japan

*Corresponding author: kyokof@toyaku.ac.jp

\section{ABSTRACT}

Detection methods for small biological molecules are needed to facilitate analysis of physiological and pathological mechanisms. We aimed to construct a 2-mercaptobenzimidazole modified gold nanoparticle electrode for detection of uremia-related substances, e.g. uric acid (UA) and indoxyl sulfate (IS), excreted via transporters expressed on cultured cells. This electrode detected the current changes in phosphate buffer at different potentials as the concentrations of ascorbic acid, UA, dopamine, and IS were linearly increased in $1 \mu \mathrm{g} / \mathrm{mL}$ increments. Real-time

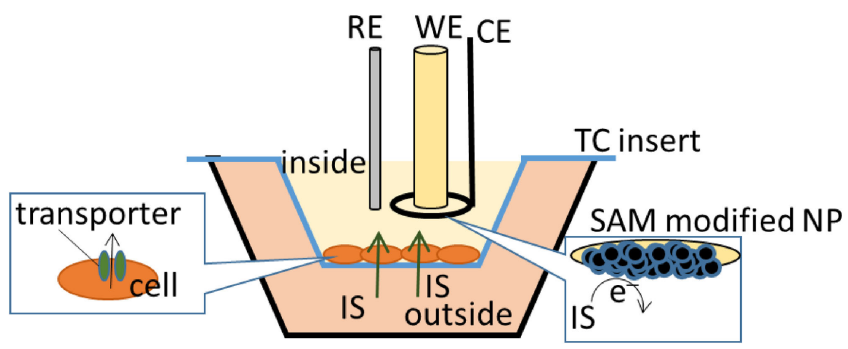
detection of IS excretion via ATP-binding cassette transporter G2 (ABCG2) expression was performed in a transcellular transport model with amperometric measurement. The electrode was highly sensitive to the current changes with IS even in a serum-free culture medium. We observed an increase in current of approximately $0.10 \mu \mathrm{A}$ per mm ${ }^{2}$ of polycrystalline electrode surface area with each $1 \mu \mathrm{g} / \mathrm{mL}$ increase in IS concentration. The current increased with time when the electrode was exposed to cells transfected with ABCG2 plasmid in tissue culture insert, indicating that IS excretion occurred via the transporter encoded by ABCG2. In conclusion, the electrode could be successfully used for the real-time detection of IS excreted via ABCG2 expressed on cultured cells.

(c) The Author(s) 2021. Published by ECSJ. This is an open access article distributed under the terms of the Creative Commons Attribution 4.0 License (CC BY http://creativecommons.org/licenses/by/4.0/), which permits unrestricted reuse of the work in any medium provided the original work is properly cited. [DOI: 10.5796/electrochemistry.21-00072].

Keywords : Modified Gold Nanoparticle Electrode, Indoxyl Sulfate, Biomolecular Detection, Transport Protein

\section{Introduction}

This paper relates to work which was awarded the Excellent Woman Researcher Award of the Electrochemical Society of Japan. Achievement of this award was based on two criteria, "development of a molecular recognition electrode" and "investigation of a biomolecular application in non-aqueous media". To fulfill the first, construction of a sensitive electrode has been investigated to

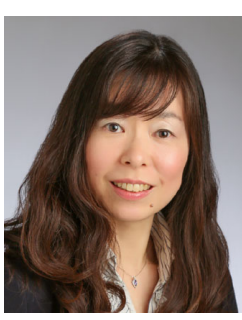

Kyoko Fujita (Senior Assistant Professor, School of Pharmacy, Tokyo University of Pharmacy and Life Sciences)

Kyoko Fujita received her $\mathrm{PhD}$ degree from Tokyo University of Agriculture and Technology (TUAT) She received a Research Fellowship for Young Scientists at JSPS. She worked as a postdoctral research fellow at Monash University and TUAT. She has been working in Tokyo University of Pharmacy and Life Sciences since 2016.

E-mail: kyokof@toyaku.ac.jp

${ }^{\S}$ ECSJ Active Member

K. Fujita (D) orcid.org/0000-0002-0871-7896 measure and understand the dynamics of uremia-related substrate in body. ${ }^{1}$ Especially, excretion of uric acid (UA) via specific transporter, the ATP-binding cassette transporter G2 (ABCG2), has been studied both in vivo using mice $^{2}$ and in vitro with cultured cells. ${ }^{3}$ To fulfill the second criterion, ionic liquids (ILs) have been studied as solvents for biomolecules. In selected "hydrated ILs" which are mixtures of IL and small amounts of water, a dissolution of biomolecules at high concentration with maintaining higher ordered structure had been reported. ${ }^{4,5}$ Dissolved biomolecules showed great thermal stability and long-term stability in hydrated ILs. ${ }^{6,7}$ Besides the biomolecular reaction, ${ }^{8,9}$ unexpected behaviors have also been reported such as dissolution and refolding of aggregated proteins in hydrated ILs. ${ }^{10,11}$ The usage of ILs is

Misaki Sekido (Undergraduate student, School of Pharmacy, Tokyo University of Pharmacy and Life Sciences)

Kohei Kanno (Undergraduate student, School of Pharmacy, Tokyo University of Pharmacy and Life Sciences)

Kio Hatae (Undergraduate student, School of Pharmacy, Tokyo University of Pharmacy and Life Sciences)

Kimiyoshi Ichida (Professor, School of Pharmacy, Tokyo University of Pharmacy and Life Sciences) 
expected to develop in the biotechnology field. In this research, we describe the electrochemical detection of uremia-related substances excreted via ATP-binding cassette transporter G2 as advanced contents of a molecular recognition electrode.

Dynamic analysis of biological materials, such as physiologically active substances, metabolites, and neurotransmitters, is important for understanding physiological and pathological mechanisms. As these small biomolecules play vital roles in the functioning of organisms, it is essential to develop detection methods for physiologically related species in biological samples. Many analytical methods have been used for the simultaneous detection of two or three physiologically related species. ${ }^{12-14}$ In analysis of multiple components, mutual response and mechanism operations should be independent. Electrochemical approaches are suitable for simultaneous detection because of their simplicity, stability, low cost, fast response time, and high sensitivity and specificity. ${ }^{15}$ Various molecular recognition electrodes for the sensitive and specific detection of biological materials have been reported. ${ }^{16-19}$

Dopamine (DA) is a neurotransmitter that plays important roles in the functioning of the central nervous, cardiovascular, and renal systems. It has been reported that oxidative stress and mitochondrial dysfunction can be improved in cells treated with DA. ${ }^{20}$ Ascorbic acid (AA) is a common antioxidant, but it is often difficult to detect or analyze it in vivo. ${ }^{21} \mathrm{UA}$ is the end product of purine metabolism in humans and is mainly excreted through the renal system. ${ }^{22}$ The importance of the extra-renal UA excretion pathway has recently attracted attention. ${ }^{23}$ We previously measured the amount of UA excreted by the intestinal lumen of rats in real time using electrochemical methods. ${ }^{2}$ To improve the observation and quantification of the excretion process, we developed a UA-sensitive electrode with a surface of self-assembled modified-monolayer gold beads. In our prior study, this electrode was used to investigate the role of the intestine in UA excretion. However, modifying this electrode with a mixture of 6-ferrocenyl-1-hexanethiol and 6hydroxy-1-hexane thiol made it difficult to detect UA separately from other biological materials, such as AA, because the detection potentials are approximately equal. Improving the electrode to enable the simultaneous detection of several biological materials would broaden its potential applications in vivo.

Uremia involves the progressive accumulation of indoxyl sulfate (IS) in blood plasma and is often associated with the progression of chronic kidney disease (CKD). IS is generally excreted in urine via the kidneys; however, with kidney failure, there is a decrease in IS excretion and an associated increase in serum IS concentration. ${ }^{24}$ Recently, the use of rat CKD models has demonstrated that IS can instead be secreted into the intestine. ${ }^{25,26}$ It was hypothesized that this transfer occurred via $\mathrm{ABCG} 2$, although this requires further clarification. In this study, we constructed an electrode to sensitively detect changes in the concentration of IS secreted via transporters expressed by cells involved in the transcellular transport system. Tissue culture (TC) inserts were used in the system, and the movement of IS from outside of insert to inside via ABCG2 was then detected electrochemically. This system was simple, as it did not require the use of labeled molecules or specialized instruments. We also aimed to investigate the selectivity of the electrode by investigating its ability to detect other biomolecules.

\section{Experiment}

\subsection{Materials}

Hydrogen tetrachloroaurate (III) tetrahydrate, trisodium citrate dihydrate, monosodium urate, dopamine hydrochloride and ascorbic acid were purchased from FUJIFILM Wako Chemical Corporation (Osaka, Japan). 2-Mercaptobenzimidazole and Indoxyl sulfate potassium salt were purchased from TCI chemicals (Tokyo, Japan) and Sigma-Aldrich Co. LLC (Tokyo, Japan), respectively. Lip- ofectamine ${ }^{\circledR} 3000$ Transfection Kit was purchased from Invitrogen by Thermo Fisher Scientific (Massachusetts, USA). All other chemicals used were commercial products of reagent or analytical grade. Polycrystalline gold electrode (diameter: $5 \mathrm{~mm}$ ) and $\mathrm{Ag} /$ $\mathrm{AgCl}-3 \mathrm{M} \mathrm{NaCl}$ electrode were purchased from BAS Inc. (Tokyo, Japan).

\subsection{Electrode preparation and biomolecules detection}

Gold nanoparticles (AuNP) were prepared according to the literature. ${ }^{27}$ Tetrachloroauric acid (III) acid solution $(1 \mathrm{mM}, 125 \mathrm{~mL})$ in a two-necked flask was incubated at $100^{\circ} \mathrm{C}$ for 1 hour. While vigorously stirring this solution, citric acid aqueous solution $(38.8 \mathrm{mM}, 12.5 \mathrm{~mL})$ was added at once, and mixed for about 20 minutes. Obtained AuNPs were measured by UV-Vis spectroscopy to calculate the particle size according to the report of Haiss et al. ${ }^{28}$ The average particle size was determined to be $15 \mathrm{~nm}$. A polycrystalline gold electrode was polish with precision abrasives 1, 3, 15 microns (Imperial Wrapping Film Sheet, 3M) until the surface becomes mirror-like, and cleaned with MilliQ. AuNP solution was concentrated 50 times by centrifuge and then dropped onto the surface of a cleaned polycrystalline gold electrode and then air dried. The drop of AuNP solution (cast) was repeated three times. ${ }^{29}$ Increase of the effective surface area with increasing cast numbers was estimated by observed current as shown in Fig. S1. Three times cast electrodes showed similar increase of effective surface area with literature. ${ }^{27}$ SAM-modified AuNP electrodes were prepared by immersing the AuNP electrodes into ethanol solutions of 2-mercaptbenzimidazole $(1 \mathrm{mM}) .{ }^{19}$ The SAM formation on the AuNP electrode was evaluated by cathodic stripping voltammetry in $0.5 \mathrm{M} \mathrm{KOH}$ solution in the potential range -0.5 to $-1.3 \mathrm{~V}$ (Fig. S2 in supporting information). In the voltammogram, two characteristic stripping peaks for 2-Mercaptobenzimidazole at -0.65 and $-1.05 \mathrm{~V}$ were observed, indicating the formation of the SAM. The SAMmodified AuNP electrode were pretreated by cyclic potential apply in $100 \mathrm{mM}$ phosphate buffer (pH 7.2) (C.E.; Pt wire, R.E.; Ag/ $\mathrm{AgCl})$. As a pretreatment, 20 or more cycles were potential swept at the same potential range $(-0.2-0.6 \mathrm{~V})$ with biomolecular detection measurement by ALS electrochemical analyzer (Model 802D, BAS Inc., Tokyo). ${ }^{30}$ The pretreated electrode was set in phosphate buffer or serum-free culture medium, then cyclic voltammograms were recorded according to addition of UA, AA, DA or IS by $1 \mu \mathrm{g} / \mathrm{mL}$. Calibration curve of the electrode was prepared based on the observed current change.

\subsection{Cell culture and gene transfection}

Human embryonic kidney cells 293 (HEK293) were cultured in Gibco Dulbecco's modified eagle medium (DMEM) supplemented with $10 \%$ FBS, $1 \%$ penicillin and streptomycin, at $37^{\circ} \mathrm{C}$ with $5 \%$ $\mathrm{CO}_{2}$ until $70-90 \%$ confluent. For the transport study, the cells were seeded onto TC inserts $(0.4 \mu \mathrm{m}$, Sarstedt). Both sides of the membrane are tissue culture treated for improved cell adhesion. Translucent membranes with a pore diameter of $0.4 \mu \mathrm{m}$ allow ideal basolateral diffusion needed for transport, secretion, diffusion and cytotoxicity studies. Lipofectamine 3000 Reagent $(2.4 \mu \mathrm{L})$ was well mixed with Opti-MEM $(60 \mu \mathrm{L}){ }^{31}$ The pcDNA-ABCG2 plasmid $(1.2 \mu \mathrm{g})$ and P3000 Reagent $(2.4 \mu \mathrm{L})$ were mixed with Opti-MEM $(60 \mu \mathrm{L})$. The Lipofectamine mixture and the plasmid mixtures were mixed together and incubated for $10 \mathrm{~min}$ at room temperature. One milliliter of this mixture was added to the culture medium in each dish. Mock cells were prepared by transfecting HEK 293 cells with the empty pcDNA 3.1 vector.

\subsection{Electrochemical detection of IS excretion through trans- porters}

Forty-eight hours after transfection, DMEM with FBS and antibiotic both in inside and outside of the TC insert were exchanged 

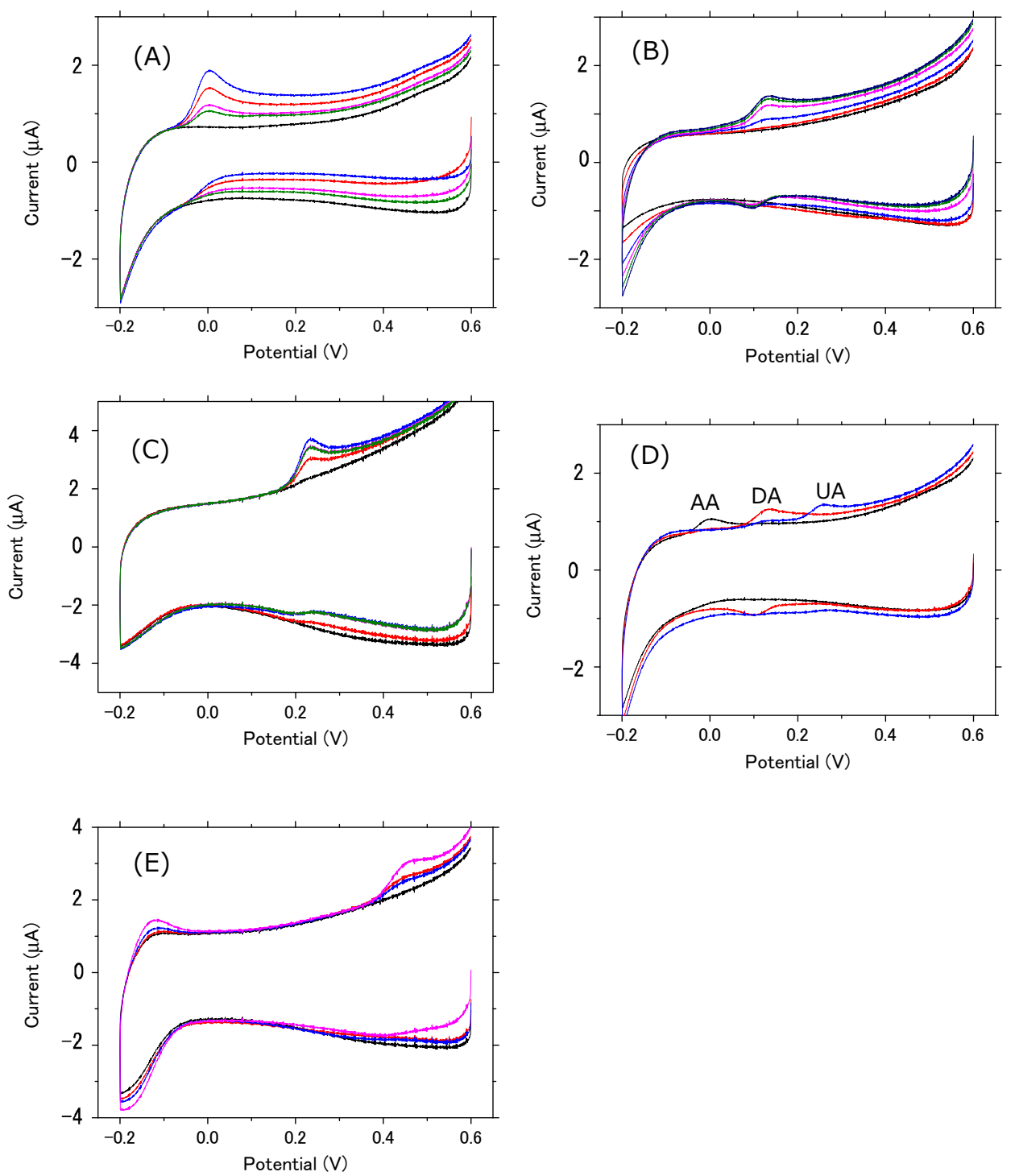

Figure 1. Cyclic voltammograms of 2-mercaptobenzimidazole modified AuNP electrode in phosphate buffer according to addition of (A) AA $(0-4 \mu \mathrm{g} / \mathrm{mL})$, (B) DA $(0-5 \mu \mathrm{g} / \mathrm{mL})$, (C) UA $(0-4 \mu \mathrm{g} / \mathrm{mL})$ or (E) IS $(0-3 \mu \mathrm{g} / \mathrm{mL})$ by $1 \mu \mathrm{g} / \mathrm{mL}$. (D) Cyclic voltammograms in phosphate buffer with $1 \mu \mathrm{g} / \mathrm{mL}$ of AA, DA and UA, respectively.

to DMEM only as a serum-free culture medium. Constructed SAMmodified AuNP electrode was set with reference and counter electrode at inside of the TC insert, and then amperometric measurement was recorded at $0.47 \mathrm{~V}$ for 40 minutes. After the first measurement, serum-free culture medium in outside of TC insert was exchanged to serum-free culture medium with $3 \mathrm{mg} / \mathrm{dL}$ IS. The second amperometric measurement was recorded for 40 minutes with the same applied potential. The correlation coefficient between measurement time and observed current value was used as change in current per second. Change in IS concentration per second was calculated by using calibration curve of each electrode. After the amperometric measurement, all cultured cells in inside of TC insert was collected and treated ultrasonic fragmentation (Bioruptor, CosmoBio. Co. LTD, Japan), then protein concentration in cultured cells was measured by using Micro BCA protein assay kit (Thermo Scientific, Massachusetts, USA).

\section{Results and Discussion}

3.1 Sensitive simultaneous detection of AA, UA, DA, and IS

The prepared 2-mercaptobenzimidazole modified AuNP electrode was immersed in phosphate buffer with $\mathrm{Pt}$ and $\mathrm{Ag} / \mathrm{AgCl}$ electrodes. The change in current in response to AA, UA, and DA concentration (Fig. 1) was measured by cyclic voltammetry (CV). The concentration of each biomolecule was increased in $1 \mu \mathrm{g} / \mathrm{mL}$ increments. For all three biomolecules, an increase in the oxidation peak current was associated with increased concentration. The observed potentials related to the oxidation peak current for AA, $\mathrm{UA}$, and DA were $0.05,0.15$, and $0.25 \mathrm{~V}$, respectively. Each potential was completely unique, as shown in Fig. 1(D). This suggested that our multi-detection platform could be used for the sensitive and simultaneous detection of AA, UA, and DA.

The change in oxidation peak current in response to the addition of IS was also measured with CV. At approximately $0.5 \mathrm{~V}$, a linear increase in oxidation peak current with increasing IS concentration was observed (Fig. 1(E)). An increase in the oxidation peak current was associated with increased IS concentration. Contrastingly, using SAM-modified AuNP electrodes without pretreatments, this linear increase in oxidation current associated with IS concentration (Fig. S3) was not observed. Therefore, the electrode pretreatments were important for obtaining stable quantitative measurements. These results indicate that the 2-mercaptobenzimidazole modified AuNP electrode sensitively detected AA, UA, DA, and IS biomolecules. 


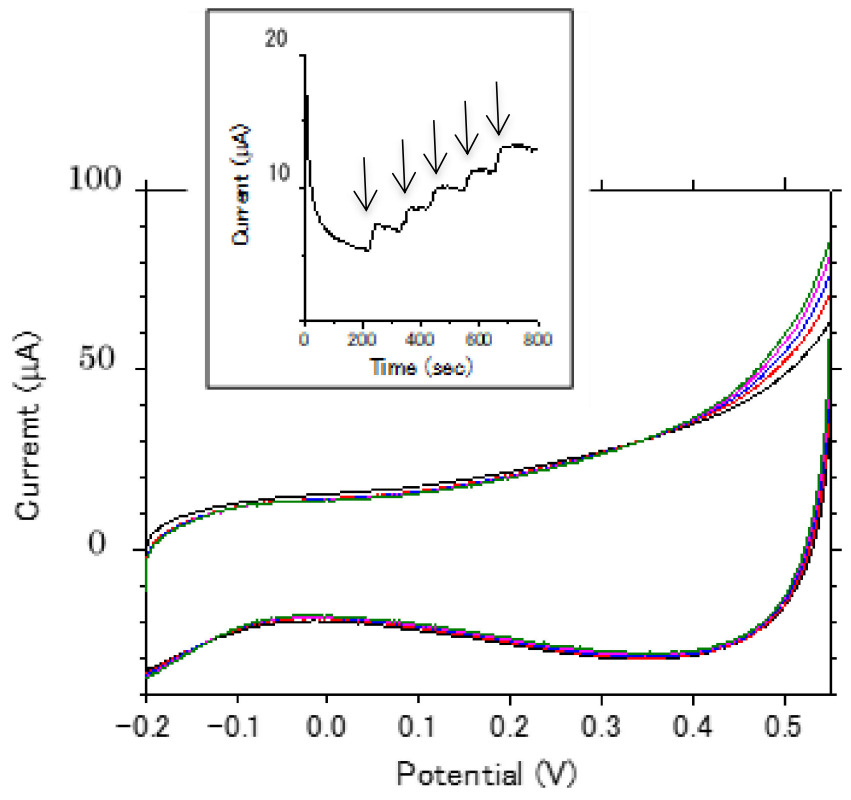

Figure 2. Cyclic voltammograms in serum-free culture medium according to addition of IS by $1 \mu \mathrm{g} / \mathrm{mL}$ increments. Insert: The faradic current change with time at approximately $0.47 \mathrm{~V}$. Addition of IS is indicated by arrows.

\subsection{Cyclic voltammetric and amperometric measurement of IS in serum-free culture medium}

Next, the measurement medium was substituted with serum-free culture medium to detect IS secreted from the ABCG2 transporter expressed on the cell culture. Figure 2 shows the cyclic voltammograms observed in response to the addition of IS to the serum-free culture medium. IS concentration was increased by $1.0 \mu \mathrm{g} / \mathrm{mL}$ increments. The faradic current at around $0.4-0.5 \mathrm{~V}$ increased linearly as the concentration of IS increased. The standard curve of the change in current versus IS concentration in serum-free culture medium is shown in Fig. S4. The 2-mercaptobenzimidazole modified AuNP electrode exhibited approximately $0.10 \mu \mathrm{A}$ increase per $\mathrm{mm}^{2}$ of polycrystalline electrode surface area with each $1 \mu \mathrm{g} / \mathrm{mL}$ change in IS concentration. The insert demonstrated an amperometric response at $0.47 \mathrm{~V}$. A stair-like increase in faradic current was observed in response to the increase in IS concentration. This suggests that real-time detection of the current in response to changes in IS concentration was possible through amperometric measurement using the 2-mercaptobenzimidazole modified AuNP electrode.

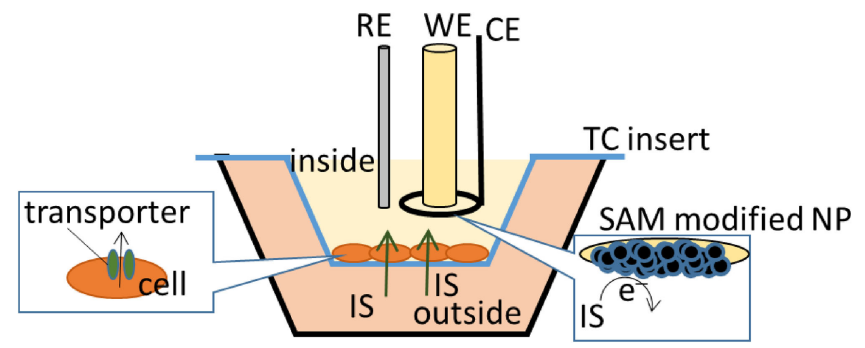

Figure 3. Schematic view of electrodes setting above cultured cell involved in the transcellular transport system using TC insert.

\subsection{Detection of IS transport via ABCG2}

Figure 3 shows the transcellular transport model used in this study. The 2-mercaptobenzimidazole modified AuNP electrode was established as the working electrode at inside of a TC insert. Counter (Pt wire) and reference $(\mathrm{Ag} / \mathrm{AgCl})$ electrodes were set with the working electrode above the transfected cell culture. The black lines in Fig. 4 show a change in the current at $0.47 \mathrm{~V}$ over time after the culture medium was replaced with serum-free culture medium. No clear change in the current was observed in either the transfected ABCG2 plasmid cells (B) or the transfected empty plasmid cells (A). The red lines in Fig. 4 show the change in current with time after the serum-free culture medium outside the TC insert was replaced with one containing $3 \mathrm{mg} / \mathrm{dL}$ IS. This IS concentration was chosen based on the serum IS level of a dialysis patient with CKD. ${ }^{32}$

In the cultured cells transfected with the empty plasmid, no critical current change was observed, suggesting that no IS was moved from outside of the TC insert to the inside. Conversely, a time-dependent current increase was observed in the cultured cells transfected with the ABCG2 plasmid. This indicated that the IS concentration within the TC insert increased and that IS secretion occurred via the ABCG2 transporter expressed on the surface of the cultured cells. The increase in IS concentration inside of the TC insert was calculated based on the gradient of the change in current with time observed with amperometric measurement. The net gradient was calculated by subtracting the change in current observed when there was no IS-containing medium outside of the insert (black line) from that including the IS-containing medium (red line). The IS excretion rate was calculated using the standard curve. The effect of the number of cultured cells was corrected for according to the protein concentration measured in each culture dish.

Figure 5 shows the box and whisker plot for the IS excretion rate of cultured cells transfected with either ABCG2 or empty plasmid $(n=10)$. While a difference in IS excretion rate was observed, this difference was not statistically significant. Overall, this suggests that
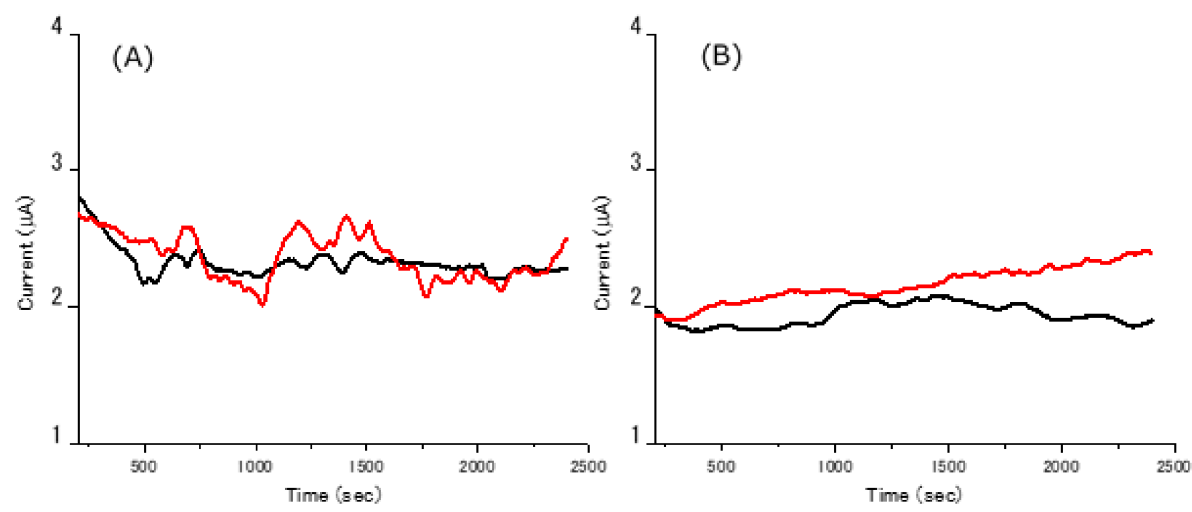

Figure 4. Current change with time when the outside of insert was filled with serum-free culture medium only (black line) and that with $3 \mathrm{mg} / \mathrm{dL}$ IS (red line). Cultured cell was transfected with (A) ABCG2 plasmid and (B) the empty plasmid. 


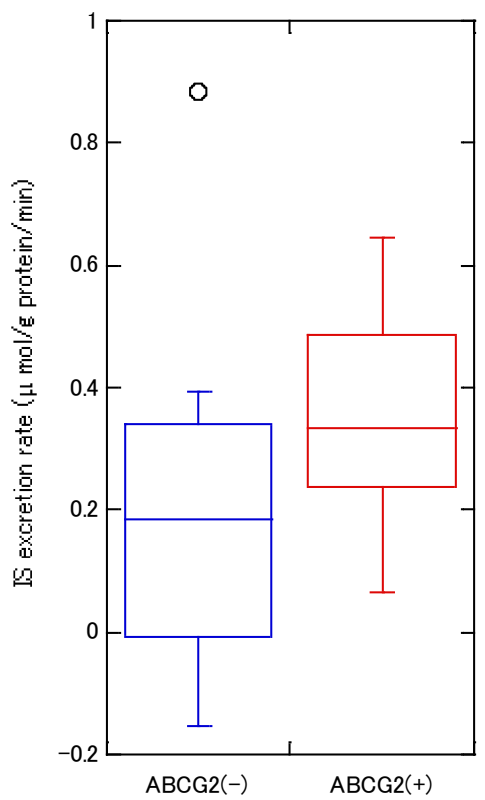

Figure 5. Box and whisker plot for the IS excretion rate of cultured cells transfected with either ABCG2 plasmid (red) or empty plasmid (blue). The box encloses $50 \%$ of the data, and the horizontal line represents the median value. The upper and lower lines indicate the upper and lower quartiles. A plot based on the cultured cells transfected with the empty plasmid shows the outliers.

the excretion of IS through the expressed ABCG2 transporter was detectable using the method described above.

\section{Conclusions}

A 2-mercaptobenzimidazole modified AuNP electrode was constructed for the detection of biological materials. After pretreatment of SAM-modified AuNP electrodes, this electrode indicated a linear change in current based on the concentration of AA, UA, DA, and IS at different potentials. This suggests that this electrode may be used as a platform for the sensitive simultaneous detection of biological materials in a transcellular transport system using a TC insert. The constructed and pretreated electrode exhibited sensitive responses to IS, even in a serum-free culture medium. The current increased by approximately $0.1 \mu \mathrm{A}$ per $\mathrm{mm}^{2}$ of polycrystalline electrode surface area with each $1 \mu \mathrm{g} / \mathrm{mL}$ change in IS concentration. An increase in current in response to IS excretion in cells transfected with $\mathrm{ABCG} 2$ plasmid was detected, supporting the findings observed in the transcellular transport model and indicating that IS excretion occurred via ABCG2. The system outlined in this paper is expected to provide an easy and sensitive method for examining the effects of external factors, such as type of drugs and the concentrations, on the secretion of IS via ABCG2.

\section{Data Availability Statement}

The data that support the findings of this study are openly available under the terms of the designated Creative Commons License in J-STAGE Data at https://doi.org/10.50892/data. electrochemistry. 15079242.

\section{References}

1. K. Fujita and K. Ichida, Clin. Chim. Acta, 463, 119 (2016).

2. K. Fujita, H. Yamada, M. Iijima, and K. Ichida, PLoS One, 14, e0226918 (2019).

3. M. Nakamura, K. Fujita, Y. Toyoda, T. Takada, H. Hasegawa, and K. Ichida, Drug Metab. Pharmacokinet., 33, 77 (2018).

4. K. Fujita, D. R. MacFarlane, and M. Forsyth, Chem. Commun., 4804 (2005).

5. K. Fujita and H. Ohno, Chem. Commun., 48, 5751 (2012).

6. K. Fujita, D. R. MacFarlane, M. Forsyth, M. Yoshizawa-Fujita, K. Murata, N. Nakamura, and H. Ohno, Biomacromolecules, 8, 2080 (2007).

7. K. Fujita, M. Sanada, and H. Ohno, Chem. Commun., 51, 10883 (2015).

8. K. Fujita and H. Ohno, Biopolymers, 93, 1093 (2010).

9. K. Fujita, N. Nakamura, K. Igarashi, M. Samejima, and H. Ohno, Green Chem., 11, 351 (2009).

10. K. Fujita, R. Nakano, R. Nakaba, N. Nakamura, and H. Ohno, Chem. Commun., 55, 3578 (2019).

11. K. Fujita, M. Kajiyama, Y. Liu, N. Nakamura, and H. Ohno, Chem. Commun., 52, $13491(2016)$

12. C.-Y. Wang, C.-W. Huang, T.-T. Wei, M.-Y. Wu, and Y.-W. Lin, Sens. Actuators, $B, \mathbf{2 4 4}, 357$ (2017)

13. Q. Wang, X. Wen, and J. Kong, Crit. Rev. Anal. Chem., 50, 359 (2020).

14. J. K. Takemoto and N. M. Davies, J. Pharm. Biomed. Anal., 54, 812 (2011).

15. M. Labib, E. H. Sargent, and S. O. Kelley, Chem. Rev., 116, 9001 (2016).

16. M. Y. Emran, M. A. Shenashen, H. Morita, and S. A. El-Safty, Biosens. Bioelectron., 109, 237 (2018).

17. L. Yang, N. Huang, Q. Lu, M. Liu, H. Li, Y. Zhang, and S. Yao, Anal. Chim. Acta, 903, 69 (2016).

18. S. A. Shahamirifard, M. Ghaedi, Z. Razmi, and S. Hajati, Biosens. Bioelectron., 114, 30 (2018)

19. C. R. Raj, F. Kitamura, and T. Ohsaka, Analyst, 127, 1155 (2002).

20. W. Duan, B. Ladenheim, R. G. Cutler, I. I. Kruman, J. L. Cadet, and M. P. Mattson, J. Neurochem., 80, 101 (2002).

21. M. A. Alonso-Lomillo, O. Domínguez-Renedo, A. Saldaña-Botín, and M. J. Arcos-Martínez, Talanta, 174, 733 (2017)

22. N. Anzai, Y. Kanai, and H. Endou, Curr. Opin. Rheumatol., 19, 151 (2007).

23. K. Ichida, H. Matsuo, T. Takada, A. Nakayama, K. Murakami, T. Shimizu, Y. Yamanashi, H. Kasuga, H. Nakashima, T. Nakamura, Y. Takada, Y. Kawamura, H. Inoue, C. Okada, Y. Utsumi, Y. Ikebuchi, K. Ito, M. Nakamura, Y. Shinohara, M. Hosoyamada, Y. Sakurai, N. Shinomiya, T. Hosoya, and H. Suzuki, Nat. Commun., 3, 764 (2012).

24. G. Lano, S. Burtey, and M. Sallée, Toxins, 12, 229 (2020)

25. T. Takada, T. Yamamoto, H. Matsuo, J. K. Tan, K. Ooyama, M. Sakiyama, H. Miyata, Y. Yamanashi, Y. Toyoda, T. Higashino, A. Nakayama, A. Nakashima, N. Shinomiya, K. Ichida, H. Ooyama, S. Fujimori, and H. Suzuki, Sci. Rep., 8, 11147 (2018).

26. K. Morimoto, Y. Tominaga, Y. Agatsuma, M. Miyamoto, S. Kashiwagura, A. Takahashi, Y. Sano, K. Yano, C. Kakinuma, T. Ogihara, and M. Tomita, Biopharm. Drug Dispos., 39, 328 (2018).

27. K. Murata, K. Kajiya, M. Nukaga, Y. Suga, T. Watanabe, N. Nakamura, and H. Ohno, Electroanalysis, 22, 185 (2010).

28. W. Haiss, N. T. Thanh, J. Aveyard, and D. G. Fernig, Anal. Chem., 79, 4215 (2007).

29. M. Suzuki, K. Murata, N. Nakamura, and H. Ohno, Electrochemistry, 80, 337 (2012).

30. R. Tanimura, M. G. Hill, E. Margoliash, K. Niki, H. Ohno, and H. B. Gray, ECS Solid State Lett., 5, E67 (2002).

31. B. Shi, M. Xue, Y. Wang, Y. Wang, D. Li, X. Zhao, and X. Li, Int. J. Physiol. Pathophysiol. Pharmacol., 10, 95 (2018).

32. F. C. Barreto, D. V. Barreto, S. Liabeuf, N. Meert, G. Glorieux, M. Temmar, G. Choukroun, R. Vanholder, and Z. A. Massy, J. Am. Soc. Nephrol., 4, 1551 (2009). 